\title{
Nanocompósitos de matriz epoxídica com reforços produzidos a partir do grafite natural
}

\author{
Epoxy resin nanocomposites with reinforcements \\ produced from natural graphite
}

Delne Domingos da Silva, Wyllian Ficagna dos Santos, Sérgio Henrique Pezzin

\author{
Universidade do Estado de Santa Catarina, Centro de Ciências Tecnológicas, \\ Programa de Pós-Graduação em Ciências e Engenharia de Materiais, \\ PGCEM/CCT/UDESC, cep 89219-710, Joinville, SC. \\ e-mail: delne.ds@gmail.com ; wyllian.f@gmail.com ; pezzin@joinville.udesc.br
}

\begin{abstract}
RESUMO
O grafite é a fonte mais abundante e de baixo custo para obtenção de grafeno. Para sua produção e aplicação em nanocompósitos em escala industrial, o método de redução do óxido de grafite (OG) tem sido o mais utilizado. A oxidação do grafite promove a introdução de grupos funcionais na sua estrutura lamelar que causam o afastamento dos planos cristalinos do grafite, produzindo assim o OG. Sua redução pode ser realizada tanto por métodos químicos quanto térmicos, a qual remove parcialmente os grupos funcionais introduzidos na oxidação, fazendo com que a estrutura grafítica seja também parcialmente reestabelecida. Várias matrizes poliméricas estão sendo utilizadas na produção de nanocompósitos com grafeno, dentre elas as resinas epoxídicas. Um dos desafios é proporcionar a dispersão total do nanoreforço na matriz e promover uma forte adesão interfacial matriz/nanoreforço para se obter melhores propriedades finais. Portanto, o objetivo deste trabalho foi caracterizar as propriedades morfológicas, mecânicas e térmicas de nanocompósitos de matriz epoxídica à base de éter diglicidílico do bisfenol A (DGEBA) com reforços produzidos a partir do grafite natural, sendo estes o grafite sonificado (GS), OG e o OG expandido (OGE) na concentração de $0,1 \% \mathrm{~m} / \mathrm{m}$. O sistema contendo o OGE se mostrou o mais promissor na melhoria das propriedades mecânicas de sistemas com resina epoxídica, uma vez que apresentou um incremento de $\sim 37 \%$ na resistência à tração. Não foram observadas alterações significativas na estabilidade térmica dos nanocompósitos, indicando que não se formam redes de percolação na concentração de reforço estudado.
\end{abstract}

Palavras-chave: Grafite, grafeno, óxido de grafite, nanocompósitos, resina epoxídica.

\section{ABSTRACT}

Graphite is the cheapest and the most abundant source to obtain graphene. For its production and application in nanocomposites in industrial scale, reduction of the graphite oxide (GO) has been the most used method lately. Graphite oxidation promotes the insertion of functional groups in its layers which lead to an increase in graphite interlayer distance, producing GO. The GO can be reduced either by chemical or thermal methods, which partially removes the functional groups inserted during oxidation, partially reestablishing the graphitic structure. Several polymer matrices have been utilized to produce graphene nanocomposites, including the epoxy resins. One of the challenges to produce polymer nanocomposites is the total dispersion of the nanofillers into the matrix and a strong matrix/nanofillers interfacial adhesion to obtain enhanced final properties. Therefore, the aim of this work was the characterization of morphological, mechanical and thermal properties of epoxy matrix based on diglycidyl ether of bisphenol-A (DGBEA) nanocomposites with reinforcements produced from natural graphite, such as sonicated graphite (SG), GO and expanded GO (EGO) at the concentrations of $0.1 \mathrm{wt} \%$. The most promising system to enhance mechanical properties of epoxy resin nanocomposites was the EGO system, since it showed an increase of $\sim 37 \%$ in tensile strength. The results showed no significant change on thermal stability of the nanocomposites indicating the absence of a percolation network with the amount of reinforcement studied.

Keywords: Graphite, graphene, Graphite oxide, Nanocomposites, Epoxy resin. 


\section{INTRODUÇÃO}

Nanocompósitos poliméricos são materiais de engenharia constituídos de um ou mais tipos de reforços, orientados ou não, com tamanhos menores do que $100 \mathrm{~nm}_{2}$ em pelo menos uma das dimensões [1]. Os nanocompósitos poliméricos têm atraído bastante interesse científico e tecnológico nos últimos anos, pois mostram melhorias substanciais nas suas propriedades mecânicas, físicas e térmicas com a adição de pequenas quantidades de nanoreforço, reduzindo o peso final, simplificando muitas vezes o processo e criando novas aplicações para os polímeros $[1,2]$. As propriedades dos nanocompósitos poliméricos dependem fortemente de como o nanoreforço está disperso na matriz [3] e isto depende de fatores como tamanho do reforço, forma, orientação e concentração na matriz [4]. Entretanto, existem dois requisitos críticos para obtenção de nanocompósitos com propriedades ótimas: dispersão do reforço na matriz e adesão interfacial matriz/reforço [5]. Sendo assim, os nanoreforços apresentam vantagens em relação aos outros tipos de reforços por possuírem maior área específica e alta razão de aspecto, favorecendo a adesão interfacial [6], embora possuam também uma grande tendência de formar aglomerados, o que prejudica sua dispersão e conseqüentemente suas propriedades finais [4]. Ao se dispersar um reforço estruturado em camadas (ex.: sílica, grafite) em matriz polimérica, pode-se obter três tipos de configurações: aglomerado, intercalado e esfoliado. Esta classificação foi primeiramente desenvolvida para exemplificar a dispersão de nanoargilas, porém tem sido também amplamente utilizada para o grafeno, óxido de grafite, entre outros [7]. Aglomerados não são desejados, uma vez que, além de terem suas áreas superficiais bastante reduzidas, geram pontos de concentração de tensão e tendem a fragilizar o material. Portanto, o objetivo é obter a esfoliação completa ou a intercalação, assim, este material terá maior área superficial efetiva, proporcionando uma melhor interação matriz/reforço e conseqüentemente maior transferência de carga.

Existem algumas técnicas citadas na literatura que visam melhorar a dispersão do nanoreforço na matriz, dentre as quais a sonificação é uma das mais utilizadas $[1, \underline{3]}$. A sonificação consiste em gerar pulsos de ultrassom de alta potência para promover a separação das nanopartículas e mantê-las em suspensão na matriz. As condições ótimas de tempo e potência utilizadas devem ser estudadas para cada sistema, de modo a se obter os melhores resultados. A adição de solventes nesta etapa pode promover a diminuição da viscosidade da matriz polimérica e conseqüentemente melhorar a dispersão das nanopartículas, uma vez que estas podem se difundir mais facilmente. Entretanto, se faz necessária a adição de mais uma etapa no processo de produção para remoção do solvente, além de que, mesmo em quantidades muito pequenas, a presença de solvente pode prejudicar as propriedades finais do compósito [3]. No caso da utilização de resina epoxídica, solventes como acetona, tetrahidrofurano (THF) e dimetilformamida (DMF) são bastante utilizados. Com relação à adesão interfacial matriz/reforço, é importante ressaltar que esta irá promover a transferência de propriedades do reforço para a matriz e vários fatores podem interferir nesse mecanismo, como polaridade, massa molecular, hidrofobicidade, grupos reativos, entre outros; presentes no polímero, reforço e/ou solvente [8]. Desta maneira, a partir da funcionalização (introdução de grupos funcionais na estrutura do reforço ou da matriz) pode-se promover a melhoria desta adesão e conseqüentemente das propriedades finais. Por exemplo, a funcionalização do grafeno pode ser obtida quando produzido a partir do óxido de grafite $(\mathrm{OG})$, uma vez que a introdução de grupos funcionais durante a oxidação, além de evitar a aglomeração das camadas, pode formar ligações covalentes na interface matriz/grafeno, melhorando também a sua dispersão [4].

As resinas epoxídicas são caracterizadas pela presença de pelo menos dois anéis epóxidos, sendo que as resinas comerciais mais estudadas são à base de diglicidil éter de bisfenol A (DGEBA), um pré-polímero de baixa massa molecular $[9,10]$. As resinas epoxídicas são convertidas em polímeros termorrígidos por uma reação de cura através da ação de endurecedores, na qual a resina é transformada em um polímero de massa molecular infinita devido à formação de ligações cruzadas, gerando uma rede tridimensional complexa. Estas mudanças ocorrem irreversivelmente por intermédio de uma reação química exotérmica, podendo ser iniciada pelo uso de diversos endurecedores, como por exemplo as aminas (alifáticas e aromáticas). Durante o processo de cura, os grupos amina interagem com os anéis epóxidos, rompendo a ligação covalente entre os átomos de oxigênio e carbono do anel, que por sua vez se ligam covalentemente ao átomo de nitrogênio presente no endurecedor, formando as ligações cruzadas [10]. As propriedades finais da resina curada dependem da estrutura química da resina e do agente de cura, bem como das condições térmicas durante o processo de cura [11]. De modo geral, as resinas epoxídicas possuem excelentes propriedades mecânicas e químicas como, por exemplo, alta resistência à tração e compressão, ótima resistência química a solventes, baixo índice de retração após a cura, além de baixo custo e facilidade de processamento, tornando-a amplamente utilizada em uma gama de aplicações industriais, sobretudo no setor eletrônico, automotivo, naval e aeroespacial. Entretanto, normalmente são frágeis e apresentam baixa resistência à propagação de trincas, devido à formação de polímeros altamente reticulados durante a cura. Devido a essa fragilidade, vários reforços já foram estudados na tentativa de minimizar problemas causados por essas propriedades $\mathrm{e}$ 
ainda continuam sendo tema de várias pesquisas. Dentre estes reforços, o grafeno tem se mostrado bastante promissor [12].

$\mathrm{O}$ grafeno é uma monocamada de átomos de carbono $\mathrm{sp}^{2}$ organizados em rede em uma estrutura bidimensional (2D) e é considerado o elemento estrutural básico de alguns alótropos de carbono. As propriedades do grafeno relatam altos valores de módulo de Young, tensão de ruptura, condutividade térmica, área específica, mobilidade de portadores de carga, além de outras propriedades. A fonte mais abundante de baixo custo para obtenção de grafeno é o grafite natural, pois este consiste em várias folhas de grafeno empacotadas, unidas por forças de Van der Walls. A maioria dos métodos de produção de grafeno envolve a produção de pequenas quantidades, entretanto, métodos de produção de grafeno em grandes quantidades têm permitido a sua utilização como reforço em nanocompósitos de matriz polimérica. As rotas químicas são consideradas as mais promissoras para sua produção em larga escala. Este método consiste em promover a redução do óxido de grafite $(\mathrm{OG})$, processo que utiliza uma mistura de ácidos e agentes oxidantes fortes, promovendo a introdução de grupos funcionais na estrutura do grafite, predominantemente grupos hidroxila e epóxidos. Assim, a partir do OG, pode-se então promover sua redução tanto pela utilização de agentes redutores ou por tratamentos térmicos, obtendo folhas de grafeno funcionalizadas. O grafeno tem atraído bastante interesse em pesquisas, devido à combinação da sua grande área específica, alta razão de aspecto, elevada condutividade elétrica e térmica e ótimas propriedades mecânicas [1, 13]. RAFIEE et al. [14] estudou algumas propriedades mecânicas de nanocompósitos epóxi/grafeno e obteve melhorias superiores às encontradas para nanocompósitos reforçados com nanotubos de carbono. O óxido de grafite (OG) também tem sido utilizado como reforço [3]. Pesquisas revelaram que nanocompósitos poliméricos reforçados com OG tratado quimicamente mostraram melhorias significativas nas propriedades térmicas e mecânicas [15]. A resina epoxídica pura possui baixa condutividade térmica, porém a adição de OG causou melhorias significativas nesta propriedade, obtendo resultados similares aos encontrados para sistemas com adição de nanotubos de carbono, mostrando que o OG é um bom material para dissipação de calor [8]. Neste contexto, o objetivo deste trabalho foi produzir nanocompósitos de matriz epoxídica para aplicações em componentes estruturais utilizando reforços produzidos a partir do grafite natural, a fim de caracterizar suas propriedades morfológicas, mecânicas e térmicas.

\section{MATERIAIS E MÉTODOS}

A partir do grafite natural, três tipos de reforços foram produzidos para a produção dos nanocompósitos de matriz epoxídica: grafite sonificado (GS), óxido de grafite (OG) e óxido de grafite expandido (OGE).

\subsection{Grafite sonificado}

O grafite natural (Labsynth, granulometria menor que $45 \mu \mathrm{m}, 98 \%$ de pureza e teor de cinzas de 1,0\%) foi sonificado por sonda de ultrassom (Sonics Vibration, $750 \mathrm{~W}, 20 \mathrm{kHz}$ ) em tetrahidrofurano (THF) (Cinética, $99,0 \%)_{2}$ na proporção de $10 \% \mathrm{~m} / \mathrm{m}$ com amplitude de $22 \%$ durante $60 \mathrm{~min}$ sob agitação magnética, obtendose o GS.

\section{2 Óxido de grafite}

O OG foi produzido utilizando o método de Hummers modificado [16]. Para isso, adicionou-se em um Becker $1,0 \mathrm{~g}$ de grafite natural, $23,0 \mathrm{~mL}$ de $\mathrm{H}_{2} \mathrm{SO}_{4}$ e $0,5 \mathrm{~g}$ de $\mathrm{NaNO}_{3}$, mantendo o sistema agitado e resfriado em banho de água e gelo. Após $15 \mathrm{~min}$, adicionou-se gradualmente 3,0 g de $\mathrm{KMnO}_{4}(99,0 \%)$, cuidando para que a temperatura não ultrapassasse $20^{\circ} \mathrm{C}$. Após $30 \mathrm{~min}$, retirou-se o banho de água e gelo, e manteve-se a mistura a $35^{\circ} \mathrm{C}$ sob agitação branda por $24 \mathrm{~h}$, obtendo-se um líquido altamente viscoso de coloração marromavermelhada. Posteriormente, $100 \mathrm{~mL}$ de uma solução de $\mathrm{H}_{2} \mathrm{SO}_{4}(5 \% \mathrm{~m} / \mathrm{m})$ foram adicionadas à mistura, causando elevação da temperatura até aproximadamente $100^{\circ} \mathrm{C}$, mudando para coloração amarelo-escuro com leve efervescência. Após $30 \mathrm{~min}$, foram adicionados $280 \mathrm{~mL}$ de uma solução de $\mathrm{H}_{2} \mathrm{O}_{2}(3 \% \mathrm{~m} / \mathrm{m})$ e a mistura ficou sob agitação por 45 min, a fim de reduzir o permanganato e o dióxido de manganês residual para sulfato de manganês, resultando em uma mistura de coloração amarelo-brilhante. Essa mistura foi então filtrada, com auxílio de um sistema a vácuo, em um funil de Büchner de $12,5 \mathrm{~mm}$ de diâmetro e papel filtro de permeabilidade de $55 \mathrm{~L} \cdot \mathrm{s}^{-1} \cdot \mathrm{m}^{-2}$, sendo em seguida lavada com $300 \mathrm{~mL}$ de uma solução de $\mathrm{HCl}(37 \%)$ morna $\left(\sim 60^{\circ} \mathrm{C}\right)$ a $10,0 \% \mathrm{~m} / \mathrm{m}$, a fim de remover íons metálicos. O material coletado no papel filtro foi então seco em estufa a vácuo por $24 \mathrm{~h}$ a $60^{\circ} \mathrm{C}$ para facilitar o posterior processo de lavagem, como sugerido por CHEN et al. [17]. Após a secagem, o material foi suspendido em 1,0 L de água deionizada e filtrado novamente com auxílio de um sistema a vácuo, repetindo-se este procedimento de lavagem por três vezes, totalizando $~ 96 \mathrm{~h}$ de filtração. Após o término da lavagem, o material coletado no papel filtro foi seco novamente em estufa a vácuo por $24 \mathrm{~h}$ a $60^{\circ} \mathrm{C}$, obtendo-se o OG. 


\section{3 Óxido de grafite expandido}

A expansão térmica do OG foi realizada em um forno tipo mufla. Para isso, o OG foi colocado no fundo de um Erlenmeyer de $50 \mathrm{~mL}$ com rolha de borracha, o qual foi colocado dentro do forno mufla pré-aquecido a $350^{\circ} \mathrm{C}$. Após 15 segundos dentro do forno, o material sofreu expansão, obtendo-se OGE. Vale ressaltar que a expansão do OG é provocada pela liberação de gases gerados pelo aquecimento, e, devido ao repentino aumento da pressão interna do frasco, o dispositivo utilizado para o seu fechamento pode ser removido, necessitando de atenção especial no momento da realização do experimento.

\subsection{Produção dos nanocompósitos}

Os nanocompósitos foram preparados pelo método in situ com resina epoxídica Araldite MY 750 à base de éter diglicidílico do bisfenol A (DGEBA) e curada com endurecedor Aradur HY 956 EN à base de poliamina alifática modificada, contendo $22-30 \%$ de trietilenotetramina (ambos fabricados pela Huntsman), na proporção de 100 partes de resina para 22 partes de endurecedor (sugerido pelo fabricante). O OG, OG e OGE $(0,1 \% \mathrm{~m} / \mathrm{m})$ foram primeiramente sonificados em THF $(10 \% \mathrm{~m} / \mathrm{m})$ por sonda de ultrassom (Sonics Vibration, $750 \mathrm{~W}, 20 \mathrm{kHz}$ ) com amplitude de $22 \%$ por 60 min sob agitação magnética, o qual foi adicionado à resina, sendo novamente sonificado nas mesmas condições. Após a dispersão do reforço na matriz, o sistema foi degaseificado para remoção do solvente com auxílio de um sistema a vácuo durante 2 horas a $70^{\circ} \mathrm{C}$ sob agitação magnética. Posteriormente, foi adicionado o endurecedor e, com um bastão de vidro, foi agitado manualmente por $5 \mathrm{mim}$. A moldagem dos corpos de prova foi feita por casting, no qual a resina foi vertida em moldes de silicone, já no formato desejado dos corpos de prova. Após a moldagem, os moldes foram mantidos em temperatura ambiente por 3 horas, seguido de $24 \mathrm{~h} \mathrm{a} 60^{\circ} \mathrm{C}$ em estufa (sistema de cura sugerido pelo fabricante). Vale lembrar que o processo de sonificação do sistema de resina epoxídica pura foi realizado na presença de solvente na proporção $10 \% \mathrm{~m} / \mathrm{m}$.

\subsection{Caracterização}

\subsubsection{Microscopia eletrônica de transmissão (MET)}

As análises de MET foram realizadas em um microscópio eletrônico de transmissão da Zeiss modelo LIBRA 120, equipado com filtro de energia (Zeiss Libra 120 EFTEM) operando a $80 \mathrm{kV}$. As análises foram realizadas na Universidade Estadual de Campinas (UNICAMP). Apenas as amostras de GS e OGE foram analisadas, as quais foram primeiramente suspensas em água em baixas concentrações e posteriormente gotejadas $(\sim 10 \mathrm{~mL})$ sobre a grade de suporte do equipamento.

\subsubsection{Espectroscopia no infravermelho por transformada de Fourier (FTIR)}

As análises de FTIR foram coletadas usando um espectrofotômetro Perkin-Elmer Spectrum One B, no total de 12 varreduras na região espectral de 4000 a $600 \mathrm{~cm}^{-1}$, com resolução de $4 \mathrm{~cm}^{-1}$, utilizando o acessório de reflectância total atenuada (ATR) com cristal de $\mathrm{ZnSe}$.

\subsubsection{Difração de raios $X$ (DRX)}

As análises de DRX foram obtidas em um difratômetro de raios X da marca Shimadzu, modelo XRD 6000, com fonte de radiação $\mathrm{CoK} \alpha$. As análises foram feitas com ângulo $2 \theta$ variando de 10 a $50^{\circ}$. O comprimento de onda utilizado foi de $\lambda=1,78897 \mathrm{~A}$, voltagem de $40,0 \mathrm{kV}$ e corrente de $30,0 \mathrm{~mA}$.

\subsubsection{Microscopia óptica}

As análises de microscopia óptica foram realizadas em um microscópio óptico (Olympus, CX31) por transmissão de luz, e as imagens foram capturadas por uma câmera digital acoplada ao microscópio (Lumenera Infinity 1-2C) e editadas com auxílio dos softwares Lumenera Infinity Capture e Lumenera Infinity Analyse. As amostras foram preparadas com espessura de 0,45 $\pm 0,05 \mathrm{~mm}$.

\subsubsection{Microscopia eletrônica de varredura (MEV)}

As análises de MEV foram realizadas em um microscópio eletrônico de varredura Zeiss DSM 940A a $15 \mathrm{kV}$ para analisar as superfícies de fratura das amostras. As amostras foram recobertas com uma camada de ouro de $\sim 25 \mathrm{~nm}$ com auxílio de um metalizador Bal-Tec SCD 050 . 


\subsubsection{Análise termogravimétrica (TGA)}

As análises de TGA foram realizadas em um equipamento TGA Q50 V20.10 (TA Instruments, USA). As amostras foram pesadas $(\sim 10 \mathrm{mg})$, colocadas em um porta amostra de platina e aquecidas da temperatura ambiente até $550^{\circ} \mathrm{C}$, a uma taxa de aquecimento de $10^{\circ} \mathrm{C} \cdot \mathrm{min}^{-1}$, sob atmosfera inerte com fluxo de nitrogênio de $50 \mathrm{~mL} \cdot \mathrm{min}^{-1}$.

\subsubsection{Calorimetria exploratória diferencial (DSC)}

As análises de DSC foram conduzidas em um equipamento DSC Q20 V24.4 (TA Instruments, USA). As amostras foram pesadas $(\sim 10 \mathrm{mg})$ e submetidas a dois aquecimentos a taxa de $10{ }^{\circ} \mathrm{C} \cdot \mathrm{min}^{-1}$, sendo o primeiro da temperatura ambiente até $200^{\circ} \mathrm{C}$, seguido de isoterma a $200^{\circ} \mathrm{C}$ por $2 \mathrm{~min}$ e, posteriormente, um resfriamento rápido até $0^{\circ} \mathrm{C}$, com isoterma nesta temperatura de $2 \mathrm{~min}$; seguido do segundo aquecimento de $0^{\circ} \mathrm{C}$ até $200^{\circ} \mathrm{C}$, ambos sob atmosfera inerte com fluxo de nitrogênio de $20 \mathrm{~mL} \cdot \mathrm{min}^{-1}$.

\subsubsection{Ensaio de tração}

Os corpos de prova de tração foram ensaiados em uma máquina universal de ensaios mecânicos EMIC DL 30000 de acordo com a norma ASTM D638M-93, utilizando-se uma célula de carga de 1000 kgf e velocidade de ensaio de $5 \mathrm{~mm} \cdot \mathrm{min}^{-1}$.

\subsubsection{Microdureza Vickers}

As análises de microdureza Vickers foram executadas em um microdurômetro digital HMV-2T Shimadzu, realizando-se 5 medidas para cada amostra com a aplicação de uma carga de 25 g por 10 segundos.

\section{RESULTADOS E DISCUSSÕES}

\subsection{Microscopia eletrônica de transmissão (MET)}

A Figura 1 mostra as imagens obtidas por microscopia eletrônica de transmissão (MET) que foram realizadas nas amostras de grafite sonificado e OGE. Na amostra de grafite sonificado notou-se que os flocos possuem aparentemente um maior número de camadas empacotadas, revelado pela coloração mais escura nas imagens. Quando comparada com as amostras de OGE, observa-se que a morfologia grafítica foi alterada, devido ao aparecimento de dobras e rugosidades. Esses resultados estão de acordo com os obtidos por MEV, no qual também foi possível observar as mesmas alterações na superfície das amostras. Nota-se que o nível de esfoliação por expansão térmica foi elevado, uma vez que as folhas observadas são bastante translúcidas, indicando a existência de poucas camadas empacotadas.GRAYFER et al. (2011) [27] obteve imagens semelhantes para o óxido de grafite expandido a $900^{\circ} \mathrm{C}$, indicando que não é necessário a utilização de temperaturas muito elevadas para esfoliar o OG. KIM et al. (2009) [28] também obteve imagens de MET muito semelhantes com o OGE.

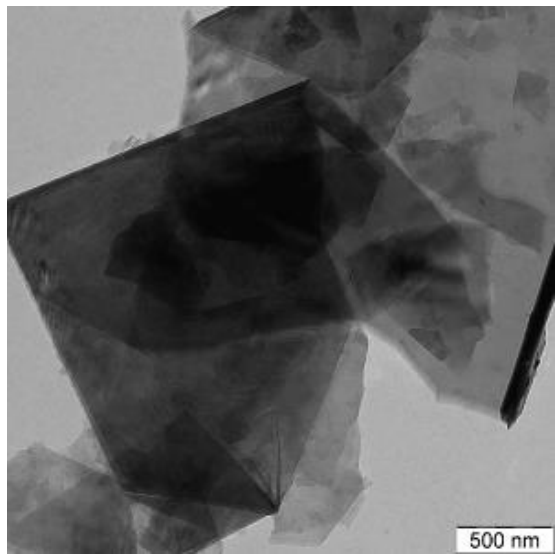

(a)

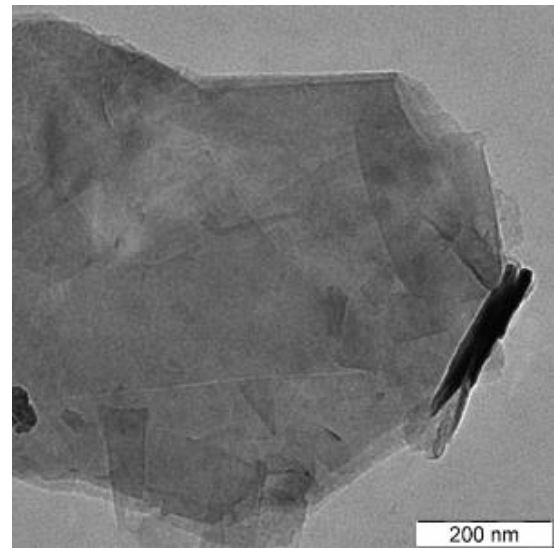

(b) 


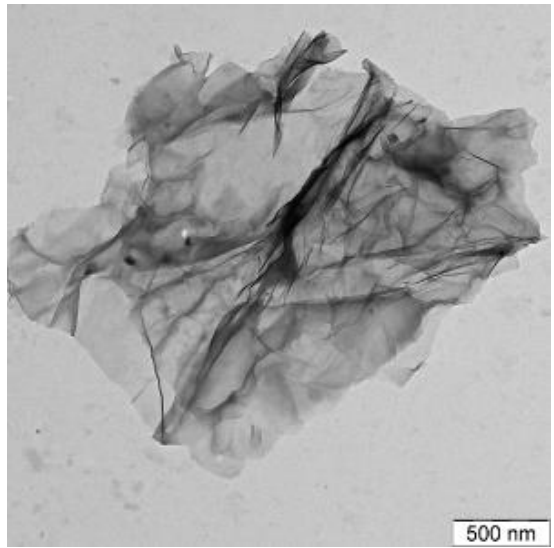

(c)

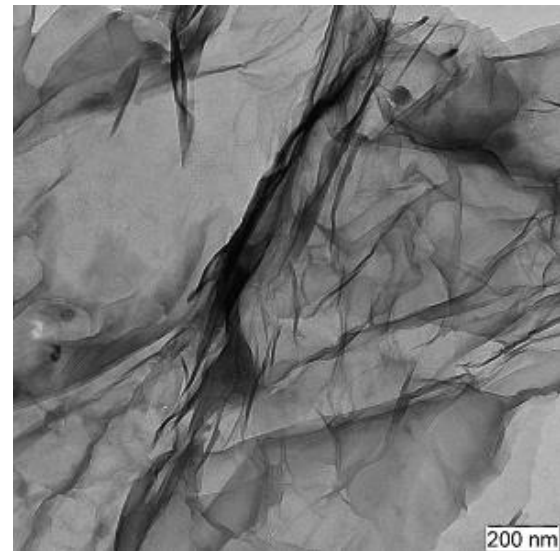

(d)

Figura 1: Imagens obtidas por MET das amostras de GS (a e b) e OGE (c e d).

\subsection{Espectroscopia no infravermelho por transformada de Fourier (FTIR)}

A Figura 2 mostra os espectros de FTIR dos sistemas de resina epoxídica pura, antes e após cura, e dos sistemas com adição de GS, OG e OGE, com detalhe mostrando a ampliação da região de absorção de 1000 a $850 \mathrm{~cm}^{-1}$, na qual é possível visualizar mais facilmente a região de absorção próxima a $915 \mathrm{~cm}^{-1}$, uma vez que esta banda é atribuída à presença de anéis epóxidos e indica o grau de cura da resina epoxídica [18]. Portanto, quanto maior a quantidade de anéis epoxídicos reagidos, menor é a intensidade dessa banda e maior o grau de cura da resina. Nota-se que a intensidade dessa banda para o espectro da resina epoxídica pura não curada é intensa e após cura praticamente desaparece, indicando um elevado grau de cura para todos os sistemas. Porém, observa-se também que a adição dos reforços causou um leve aumento na intensidade dessa banda, podendo indicar que os reforços dificultam a reação dos grupos epóxidos. Outras bandas importantes na interpretação de espectros de resina epoxídica estão em $1242 \mathrm{~cm}^{-1}$, atribuída à deformação axial simétrica em fase das ligações $\mathrm{C}-\mathrm{C}$ e $\mathrm{C}-\mathrm{O}$ de anéis epóxidos, na qual essas ligações se estiram e contraem em fase, e em $864 \mathrm{~cm}^{-1}$, atribuída à deformação axial assimétrica do anel epóxido, na qual as ligações $\mathrm{C}-\mathrm{C}$ não se alteram, enquanto uma ligação $\mathrm{C}-\mathrm{O}$ estira e a outra contrai.

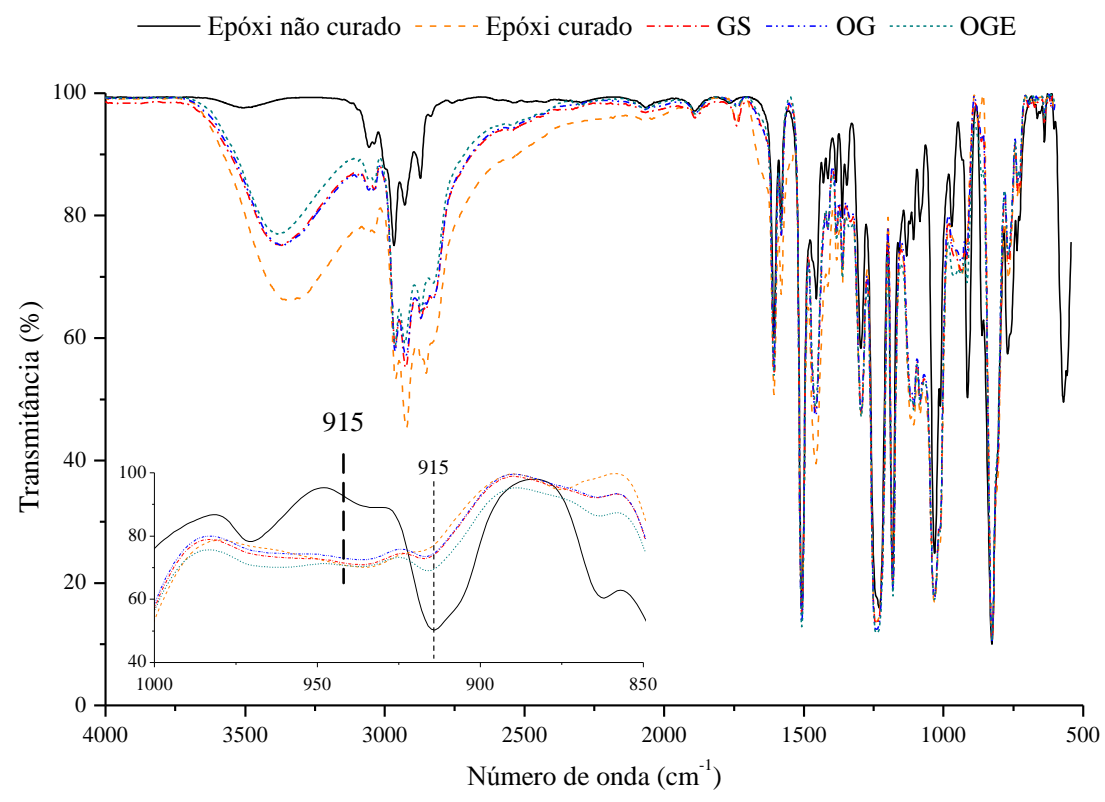

Figura 2: Espectros no FTIR na região de absorção de 4000 a $600 \mathrm{~cm}^{-1}$ dos sistemas de resina epoxídica pura, antes e após cura, e dos sistemas com adição de $0,1 \% \mathrm{~m} / \mathrm{m}$ de GS, OG e OGE. No detalhe, ampliação da região de absorção de 1000 a $850 \mathrm{~cm}^{-1}$. 
Tabela 1: Principais bandas de absorção da resina epoxídica pura não curada, curada com e sem sonificação, e dos sistemas de resina epoxídica com adição de GS, OG e OGE.

\begin{tabular}{|c|c|}
\hline BANDAS (CM-1) & ATRIBUIÇÕES \\
\hline 3384 & Deformação axial da ligação O-H \\
\hline 3038 & Deformação axial da ligação $\mathrm{C}-\mathrm{H}$ de anéis aromáticos \\
\hline 2963,2925 e 2871 & Deformação axial da ligação $\mathrm{C}-\mathrm{H}$ de grupos $\mathrm{CH}_{2} \mathrm{e} \mathrm{CH}_{3}$ \\
\hline 1607,1581 e 1507 & Deformação axial da ligação C-C de anéis aromáticos \\
\hline 1457 & Deformação angular assimétrica da ligação $\mathrm{C}-\mathrm{H}$ de grupos $\mathrm{CH}_{3}$ \\
\hline 1362 & Deformação angular simétrica da ligação $\mathrm{C}-\mathrm{H}$ de grupos $\mathrm{CH}_{3}$ \\
\hline 1295 & Deformação angular das ligações $\mathrm{C}-\mathrm{H}$ de anéis aromáticos \\
\hline 1242 & $\begin{array}{l}\text { Deformação axial simétrica em fase das ligações } \mathrm{C}-\mathrm{C} \text { e } \mathrm{C}-\mathrm{O} \text { de anéis epóxidos, na qual } \\
\text { essas ligações se estiram e contraem em fase }\end{array}$ \\
\hline 1181 e 1033 & Deformação axial da ligação $\mathrm{C}-\mathrm{O}$ acoplada à deformação axial da ligação $\mathrm{C}-\mathrm{C}$ adjacente \\
\hline 915 & $\begin{array}{l}\text { Deformação axial assimétrica do anel epóxido, na qual as ligações } \mathrm{C}-\mathrm{C} \text { estiram, enquanto } \\
\text { as ligações } \mathrm{C}-\mathrm{O} \text { se contraem }\end{array}$ \\
\hline 864 & $\begin{array}{l}\text { Deformação axial assimétrica do anel epóxido, na qual as ligações C-C não se alteram, } \\
\text { enquanto uma ligação C-O estira e a outra contrai }\end{array}$ \\
\hline 826 & Deformação angular simétrica fora do plano do grupo $=\mathrm{CH}_{2}$ \\
\hline 767 e 736 & Deformação angular fora do plano da ligação $\mathrm{C}-\mathrm{H}$ de anéis aromáticos \\
\hline
\end{tabular}

A Tabela 1 mostra as atribuições das principais bandas de absorção observadas nos espectros de FTIR referentes à resina epoxídica pura curada e não curada [18]. Não foram observadas bandas de absorção referentes ao solvente utilizado na etapa de sonificação, ou seja, não se observa a presença de THF residual após cura, mostrando que a etapa de extração de solvente por degaseificação foi eficiente. Não foram observadas diferenças significativas entre os espectros, não sendo evidenciada a formação de ligações covalentes entre matriz/reforço, resultado esperado devido à baixa concentração do reforço na matriz.

\subsection{Difração de raios $X(D R X)$}

As análises de DRX (Figura 3) revelam que todos os sistemas apresentaram estrutura semelhante, com a presença de um halo amorfo largo e de baixa intensidade aproximadamente entre os ângulos $2 \theta$ de 15 e $30^{\circ}$. Apenas nos sistemas contendo GS foi possível observar um pico cristalino de baixa intensidade em $30,9^{\circ}$, que está associado ao plano cristalino (002), característico do grafite ( $d=0,34 \mathrm{~nm})$ [19]. YASMIN et al. [20] também estudou por DRX diferentes tipos de dispersão do reforço em matriz epoxídica em compósitos com adição de $1,0 \% \mathrm{~m} / \mathrm{m}$ de grafite expandido. Os resultados encontrados mostraram que nenhuma das técnicas foi capaz de esfoliar e separar as camadas de grafite completamente, porém foram detectadas diferenças na intensidade dos picos de cristalinidade referente ao grafite, sendo que a maior intensidade foi obtida na amostra dispersada por sonda de ultrassom. As outras técnicas estudadas (banho de ultrassom, calandra e a combinação das duas) mostraram intensidade de picos menores, indicando uma dispersão mais uniforme do grafite na matriz. Não foi possível evidenciar diferenças entre os sistemas contendo OG e OGE. De acordo com as análises realizadas nos reforços puros, o pico referente ao OG deveria estar em $\sim 13^{\circ}(d=0,77 \mathrm{~nm})$, sugerindo que o reforço está bem disperso na matriz. Como o OGE não possui nenhum pico cristalino para sua identificação, não foi possível avaliar sua dispersão pela técnica de DRX. 


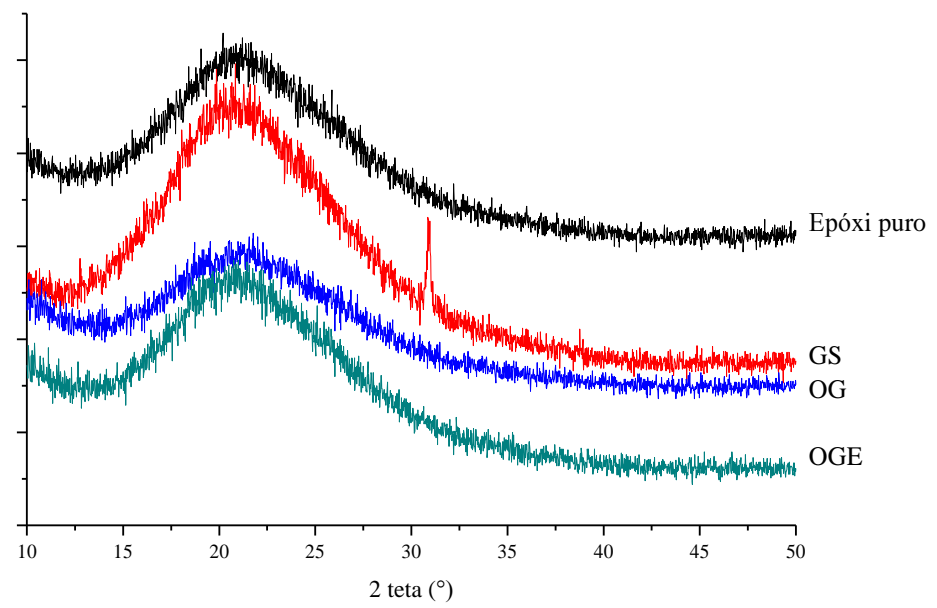

Figura 3: Difratogramas de raios $X$ do sistema curado de resina epoxídica pura e dos sistemas com adição de $0,1 \% \mathrm{~m} / \mathrm{m}$ de GS, OG e OGE.

\subsection{Microscopia óptica}

As análises de microscopia óptica revelam a dispersão do reforço na matriz (Figura 4). Nas imagens obtidas no sistema com adição de GS é possível observar a presença de grandes aglomerados, ou flocos de grafite, indicando baixa dispersão do GS na matriz. Observando as imagens do sistema com OG, nota-se também a presença de aglomerados, porém aparentemente de dimensões menores quando comparados com os sistemas com GS. Como o OG possui um maior espaçamento interplanar (confirmado por DRX) e as forças de Van der Waals são enfraquecidas, o processo de dispersão do reforço na matriz é facilitado. Nota-se também que a coloração dos flocos é diferenciada, mais próxima ao marrom e apresentando certa transparência, o que indica que a espessura dos aglomerados é menor. Já nas imagens obtidas do sistema com OGE, observa-se uma maior quantidade de aglomerados quando comparados aos sistemas com GS e OG, porém melhor dispersos na matriz e de tamanhos menores. Estes resultados podem ser explicados devido ao OGE possuir maior área superficial, aumentando sua interação com a matriz e melhorando sua dispersão, embora também possua grande tendência de se aglomerar.

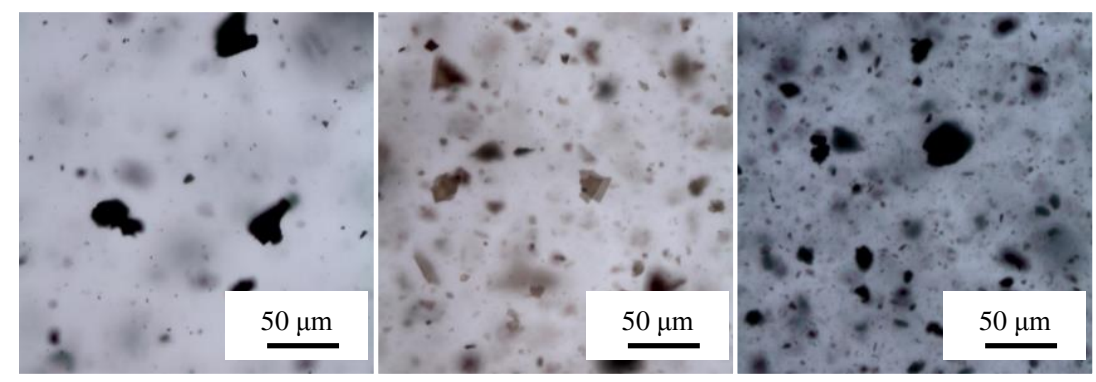

Figura 4: Imagens obtidas por microscopia óptica dos sistemas curados de resina epoxídica com adição de $0,1 \% \mathrm{~m} / \mathrm{m}$ de (a) GS, (b) OG e (c) OGE.

\subsection{Microscopia eletrônica de varredura (MEV)}

As análises de MEV foram realizadas para avaliar a morfologia da superfície de fatura dos corpos de prova submetidos ao ensaio de tração, as quais estão mostradas na Figura 5. Todas as amostras analisadas apresentaram aspecto de fratura frágil, característico das resinas epoxídicas. Observando as micrografias dos sistemas com adição de GS, nenhuma alteração na morfologia de fratura foi observada, embora algumas regiões sugeriram a presença de aglomerados. A visualização desses aglomerados se torna difícil nos sistemas com GS devido ao grafite possuir uma estrutura lamelar que se confunde com a própria matriz. Nas micrografias dos sistemas com OG também não foi possível observar alterações na morfologia de fratura, porém algumas regiões indicam a presença de aglomerados. Assim como nos sistemas com GS, a visualização dos aglomerados de OG também é dificultada devido à sua estrutura lamelar, somado ao fato de possuir uma estrutura funcionalizada, a qual melhora sua dispersão e interação matriz/reforço, dificultando sua visualização por MEV [12]. Nos sistemas com adição de OGE, a visualização dos aglomerados é 
facilitada devido à grande tendência do OGE se empacotar. Uma vez que possui grande área superficial e pelas fortes forças de Van der Walls, a sua dispersão na matriz é dificultada, formando aglomerados.
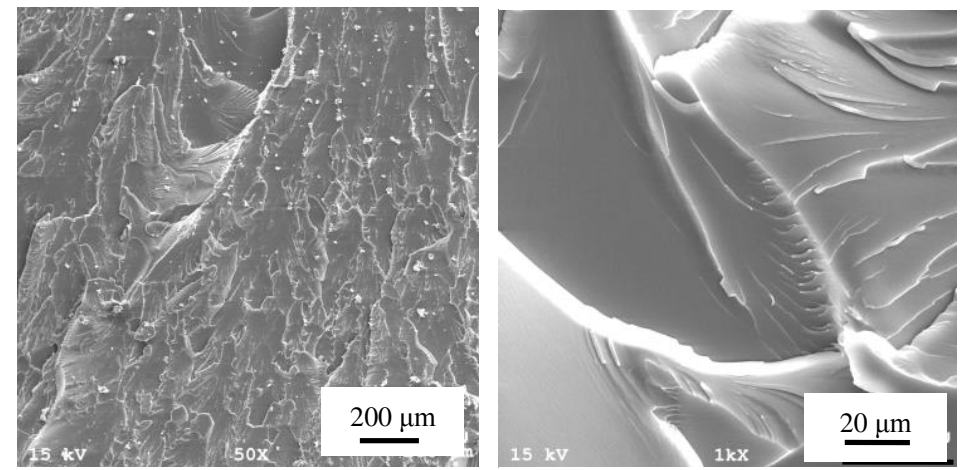

(a)
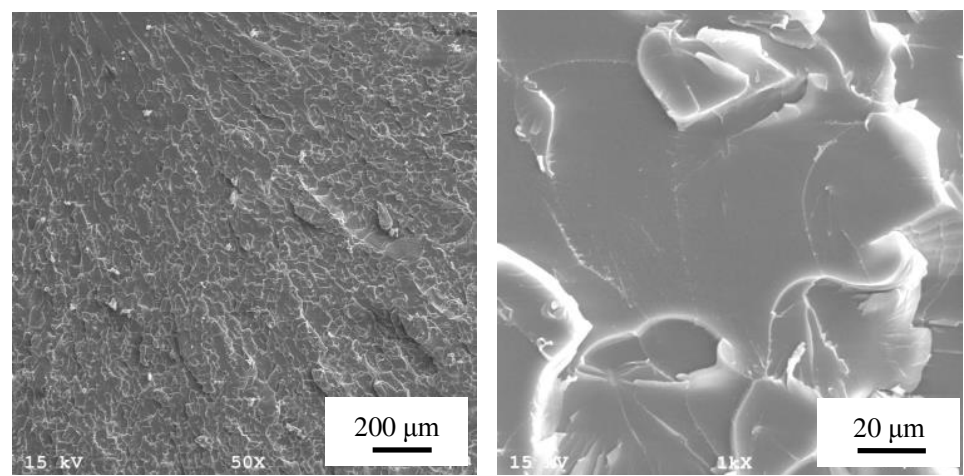

(b)
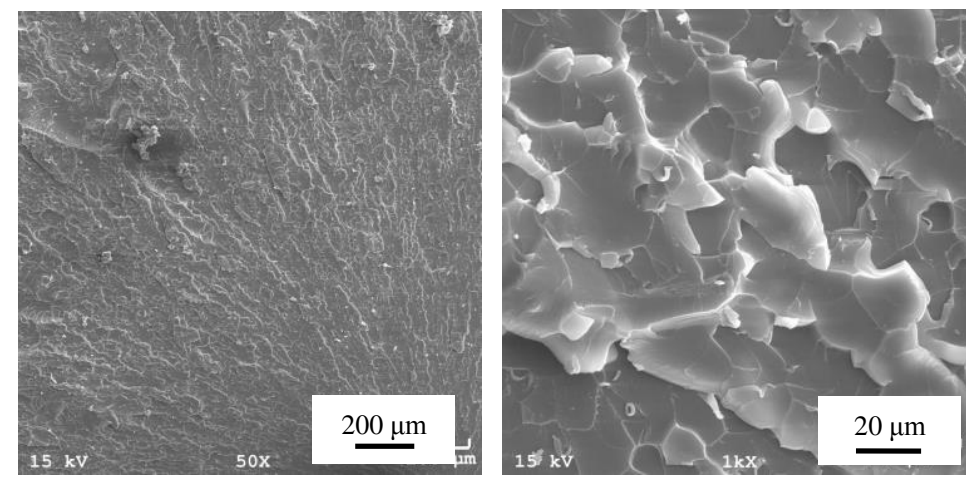

(c)
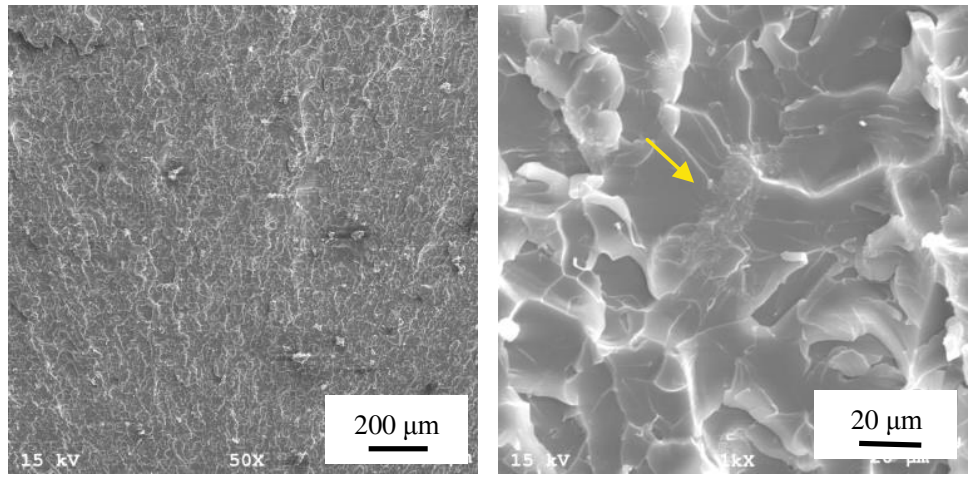

(d)

Figura 5: Micrografias da superfície de fratura do sistema curado de (a) resina epoxídica pura e dos sistemas com adição de $0,1 \% \mathrm{~m} / \mathrm{m}$ de (b) GS, (c) OG e (d) OGE. 


\subsection{Análise termogravimétrica (TGA)}

A Tabela 2 mostra os valores das temperaturas de início de perda de massa $\left(T_{\text {onset }}\right)$ e o ponto em que a taxa de perda de massa é máxima $\left(\mathrm{T}_{\text {peak }}\right)$ para os sistemas curados de resina epoxídica pura e dos sistemas com adição de GS, OG e OGE. Observou-se que para todos os sistemas com adição dos reforços, a $T_{\text {onset }}$ e a $T_{\text {peak }}$ foram um pouco menores quando comparadas ao sistema de resina pura, porém não significativas, apresentando uma redução de $\sim 1,3 \mathrm{e} \sim 1,1 \%$, respectivamente. Em todos os sistemas estudados, foi observada uma pequena perda de massa entre 50 e $275^{\circ} \mathrm{C}$, indicando a presença residual de umidade e/ou solvente, valores também apresentados na Tabela 2 . Acima de $275^{\circ} \mathrm{C}$ a perda de massa é atribuída à decomposição térmica da própria resina epoxídica. Na faixa de temperatura de 50 a $275^{\circ} \mathrm{C}$, a perda de massa média para todos os sistemas foi de apenas $2,2 \pm 0,2 \%$, sugerindo que a etapa do processo de remoção de solvente por degaseificação foi eficiente. Notou-se também uma pequena diminuição na estabilidade térmica dos sistemas com adição dos reforços quando comparados à resina pura. NGUYEN et al. [1] observou que concentrações de até $3,0 \% \mathrm{~m} / \mathrm{m}$ de OGE em matriz de poliuretano termoplástico (TPU) causam um acréscimo na $\mathrm{T}_{\text {peak }_{2}} \mathrm{e}$ que em concentrações mais altas, de 4 a $7,0 \% \mathrm{~m} / \mathrm{m}$, esta é diminuída, o que é explicado pelo fato do nanoreforço possuir alta razão de aspecto e atuar como barreira na difusão dos voláteis da decomposição. Nos sistemas estudados, esta pequena redução na estabilidade térmica causada pela adição dos reforços pode então ser atribuída a um pequeno aumento do volume livre provocado pela incorporação dos reforços, que também podem estar atuando como barreira na difusão dos voláteis.

Tabela 2: Valores de $\mathrm{T}_{\text {onset }}$ e $\mathrm{T}_{\text {peak }}$, bem como o teor residual de umidade e/ou THF, do sistema curado de resina epoxídica pura e dos sistemas com adição de $0,1 \% \mathrm{~m} / \mathrm{m}$ de GS, OG e OGE.

\begin{tabular}{l|l|l|l}
\hline & TONSET $\left({ }^{\circ} \mathbf{C}\right)$ & TPEAK $\left({ }^{\circ} \mathbf{C}\right)$ & TEOR RESIDUAL $(\%)$ \\
\hline EPÓXI PURO & 344,3 & 368,4 & 2,1 \\
\hline GS & 340,3 & 363,8 & 2,2 \\
\hline OG & 340,6 & 361,2 & 2,1 \\
\hline OGE & 339,9 & 362,0 & 2,3 \\
\hline
\end{tabular}

\subsection{Calorimetria exploratória diferencial (DSC)}

Os valores encontrados para a temperatura em que ocorre o máximo do pico endotérmico $\left(T_{t}\right)$, no primeiro aquecimento, e para a temperatura de transição vítrea $\left(\mathrm{T}_{\mathrm{g}}\right)$, obtida no segundo aquecimento a partir dos pontos de inflexão das curvas de DSC, estão na Tabela 3. Durante o primeiro aquecimento, observa-se o fenômeno de relaxação entálpica, que está relacionada às mudanças estruturais da resina epoxídica, por consequiência do envelhecimento em temperaturas abaixo da $T_{g}$. Este processo, cuja extensão depende do grau de conversão e, consequentemente, da densidade de reticulação da matriz, é indicado pelo pico endotérmico a $T_{t}$, que não aparece no segundo aquecimento, pois toda a história térmica do material foi apagada após o término do primeiro ciclo de aquecimento [21]. O processo de relaxação causa a diminuição do volume livre e da mobilidade segmental, o que explica a grande diferença entre a $T_{t}$ e a $T_{g}$, obtida no segundo aquecimento após a eliminação da história térmica da amostra. Durante o segundo aquecimento não foram observados eventos endo ou exotérmicos, sendo possível determinar o valor da $\mathrm{T}_{\mathrm{g}}$ para todas as amostras. As análises de espectroscopia no infravermelho indicaram um alto grau de cura, porém não completa, para os corpos de prova preparados pela rota proposta. Assim, além do processo de relaxação entálpica, há também a possibilidade da ocorrência de uma pós-cura causada pela temperatura mais elevada do ensaio $\left(200^{\circ} \mathrm{C}\right)$. Com base nestes resultados, sugere-se que estes sistemas sejam submetidos a uma póscura de cerca de $30 \mathrm{~min}$ em temperaturas próximas a $150^{\circ} \mathrm{C}$, a fim de assegurar melhores propriedades finais.

Nota-se que no sistema com adição de OG há uma diminuição sensível na $T_{g}$, enquanto os valores obtidos com GS e OGE oscilaram ao redor do valor encontrado para a resina pura. Segundo COSTA et al. [22], a adição de reforços em matriz polimérica pode aumentar a quantidade de microvazios, que podem ser originados de bolhas de ar aprisionadas no sistema, de bolsas de resina, de umidade absorvida durante a sua armazenagem/processamento e de ciclos de cura inadequados. A presença desses microvazios pode causar decréscimo da $T_{g}$ devido à presença de volume livre [22, 23]. Por outro lado, ZAMAN et al. [24] relatou aumento da $\mathrm{T}_{\mathrm{g}}$ de cerca de $14^{\circ} \mathrm{C}$ com adição de $2,5 \% \mathrm{~m} / \mathrm{m}$ de GS em resina epoxídica, enquanto HU et al. [25] observou um aumento na $\mathrm{T}_{\mathrm{g}}$ de $8^{\circ} \mathrm{C}$ em nanocompósitos de PS/OG reduzido quimicamente, e associou o resultado à dificuldade de movimentação das cadeias poliméricas devido à interação do polímero com o reforço, aumentando a densidade e a $T_{g}$, conseqüentemente. Na concentração de nanoreforços utilizada neste trabalho, no entanto, não foram observadas evidências dessas interações carga/matriz. 
Tabela 3: Valores de $\mathrm{T}_{\mathrm{g}}$ do sistema curado de resina epoxídica pura e dos sistemas com adição de $0,1 \% \mathrm{~m} / \mathrm{m}$ de GS, OG e OGE.

\begin{tabular}{l|l|l}
\hline & TT $\left({ }^{\circ} \mathbf{C}\right)$ & TG $\left({ }^{\circ} \mathbf{C}\right)$ \\
\cline { 2 - 3 } & $\mathbf{1}^{\circ}$ AQUECIMENTO & $\mathbf{2}^{\circ}$ AQUECIMENTO \\
\hline EPÓXI PURO & 74,5 & 104,5 \\
\hline GS & 78,5 & 108,4 \\
\hline OG & 75,2 & 84,9 \\
\hline OGE & 78,0 & 101,1 \\
\hline
\end{tabular}

\subsection{Ensaio de tração}

Os valores de resistência à tração e os módulos de Young obtidos estão apresentados na Figura 6. Nota-se que os valores de resistência à tração para os sistemas contendo GS e OG não tiveram variação significativa, porém o sistema com OGE apresentou um aumento significativo de ca. 37\%. Este aumento na resistência à tração pode ser devido à maior interação matriz/reforço quando comparado aos outros sistemas. RAFIEE et al. [12] encontraram incrementos de $\sim 40 \%$ e aumento de $\sim 45 \%$ na resistência à tração nos nanocompósitos com adição de $0,125 \% \mathrm{~m} / \mathrm{m}$ de OGE em matriz epoxídica, mostrando que os dados obtidos neste trabalho estão de acordo com outros relatados na literatura. Analisando o gráfico do módulo de Young, nota-se que a adição de GS não causou uma variação significativa, porém a adição de OG e OGE reduziu levemente os valores dos módulos. Esta diminuição pode ter sido causada pela baixa dispersão do OG e do OGE na matriz epoxídica, uma vez que a presença de aglomerados pode atuar como ponto de concentração de tensão e formação de trincas.
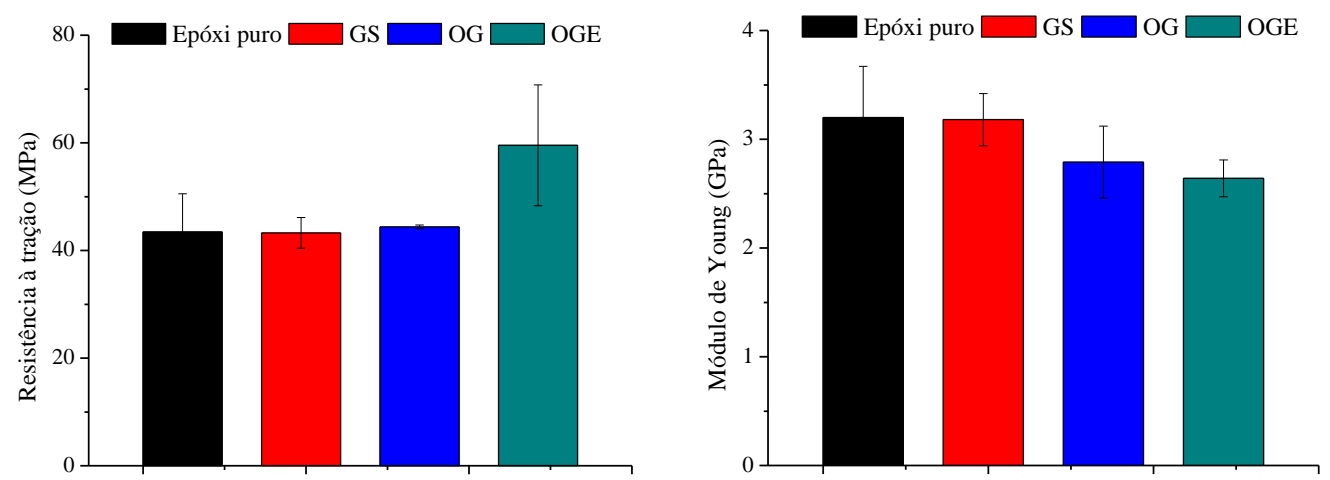

Figura 6: Gráfico dos valores de resistência à tração e dos módulos de Young dos sistemas curados de resina epoxídica pura e dos sistemas com adição de $0,1 \% \mathrm{~m} / \mathrm{m}$ de GS, OG e OGE.

\subsection{Microdureza Vickers}

O gráfico da Figura 7 mostra os valores obtidos nas análises de microdureza Vickers. Os valores de microdureza para todos os sistemas não apresentaram alterações significativas quando comparados aos sistemas de resina epoxídica pura. Observando o gráfico, nota-se uma pequena tendência de decréscimo da microdureza nos sistemas com adição OG. De acordo com COSTA et al. [22], a adição de reforços na matriz pode promover um aumento de microvazios, resultando na redução da microdureza do material. Portanto, como as variações não foram significativas, os resultados indicam que a adição dos reforços não causou aumento significativo de microvazios. SUAVE [26] encontrou valores de microdureza para nanocompósitos de matriz epoxídica/nanotubos de carbono ligeiramente inferiores à matriz pura, chegando a uma redução de cerca de $9 \%$; e associou este fato à presença de microvazios. 


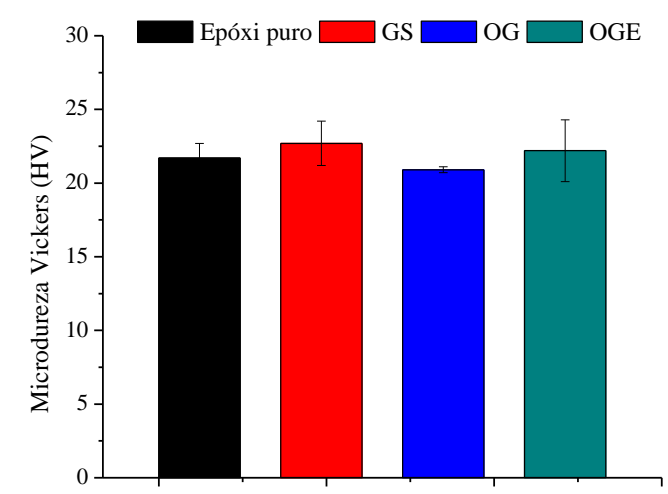

Figura 7: Gráfico dos valores de microdureza Vickers dos sistemas curados de resina epoxídica pura e dos sistemas com adição de $0,1 \% \mathrm{~m} / \mathrm{m}$ de GS, OG e OGE.

\section{CONCLUSÕES}

O OG e o OGE podem ser obtidos por métodos simples a partir do grafite natural, viabilizando sua aplicação como reforços em nanocompósitos em escala industrial. Na concentração estudada, os nanocompósitos não apresentaram alterações significativas nas propriedades térmicas, indicando que concentrações maiores de reforço devem ser utilizadas para se conhecer melhor o efeito desses reforços em matriz epoxídica. O sistema contendo o OGE se mostrou o mais promissor na melhoria das propriedades mecânicas de sistemas com resina epoxídica, uma vez que apresentou um incremento de $\sim 37 \%$ na resistência à tração.

\section{AGRADECIMENTOS}

Os autores agradecem a AEB/Programa Uniespaço, CAPES e CNPq pelo auxílio financeiro.

\section{BIBLIOGRAFIA}

[1] NGUYEN, D.A., LEE, Y.R., RAGHU, A.V., et al., "Morphological and physical properties of a thermoplastic polyurethane reinforced with functionalized graphene sheet", Polymer International, v. 58, pp. 412-417, 2009.

[2] POTTS, J.R., DREYER, D.R., BIELAWSKI, C.W., et al., "Graphene-based polymer nanocomposites", Polymer, v. 52, pp. 5-25, 2011.

[3] KIM, H., ABDALA, A.A., MACOSKO, C.W., "Graphene/polymer nanocomposites”, Macromolecules, v. 43, pp. 6515-6530, 2010.

[4] FIEDLER, B., GOJNY, F.H., WICHMANN, M.H.G., et al., "Fundamental aspects of nano-reinforced composites", Composites Science and Technology, v. 66, pp. 3115-3125, 2006.

[5] KUMAR, S.K., KRISHNAMOORTI, R., "Nanocomposites: structure, phase behavior, and properties", Annual Review of Chemical and Biomolecular Engineering, v. 1, pp. 37-58, 2010.

[6] STANKOVICH, S., DIKIN, D.A., DOMMETT, G.H.B., et al., "Graphene-based composite materials", Nature, v. 442, pp. 282-286, 2006.

[7] ALEXANDRE, M., DUBOIS, P. "Polymer-layered silicate nanocomposites: preparation, properties and uses of a new class of materials", Materials Science and Engineering, v. 28, pp. 1-63, 2000.

[8] KUILLA, T., BHADRA, S., YAO, D., et al., "Recent advances in graphene based polymer composites", Progress in Polymer Science, v. 35, pp. 1350-1375, 2010.

[9] FAZENDA, J.M.R., Tintas e vernizes: ciência e tecnologia, ABRAFATI, São Paulo-SP, 1993.

[10] DALEY, R.F., DALEY, S.J., Organic Chemistry. Daley\&Daley, 2001.

[11] MENEZES, G.V., MONTEIRO, S.N., D'ALMEIDA, et al., "Análise térmica da resina epóxi DGBEA/TETA para formulações diferentes da razão estequiométrica", Revista ABM, v. 1, pp. 12-16, 2004.

[12] RAFIEE, M.A., RAFIEE, J., WANG, Z., et al., "Enhanced mechanical properties of nanocomposites at low graphene content", ACSNano, v. 3, pp. 3884-3890, 2009.

[13] RAFIEE, M.A., LU, W., THOMAS, A.V., et al., "Graphene nanoribbon composites", ACSNano, v. 4, pp. 7415-7420, 2010. 
[14] RAFIEE, M.A., RAFIEE, J., SRIVASTAVA, I., et al., "Fracture and fatigue in graphene nanocomposites", Small, v. 6, pp. 179-183, 2010.

[15] GONG, L., KINLOCH, I.A., YOUNG, R.J., et al., "Interfacial stress transfer in a graphene monolayer nanocomposite", Advanced Materials, v. 22, pp. 2694-2697, 2010.

[16] HUMMERS, W.S., OFFEMAN, R.E., "Preparation of graphitic oxide", Journal of the American Chemical Society, v. 80, pp. 1339, 1958.

[17] CHEN. T., ZENG, B., LIU, J.L., et al., "High throughput exfoliation of graphene oxide from expanded graphite with assistance of strong oxidant in modified hummers method", Journal of Physics: Conference Series, v. 188, pp. 1-5, 2009.

[18] SILVERSTEIN, R.M., WEBSTER, F.X., Identificação espectrométrica de compostos orgânicos, 6 ed. LTC, Rio de Janeiro, 2000.

[19] STANJEK, H., HÄUSLER, W., “Basics of X-ray diffraction”, Hyperfine Interactions, v. 154, pp. 107-119, 2004.

[20] YASMIN, A., LUO, J-J; DANIEL, I.M., "Processing of expanded graphite reinforced polymer nanocomposites”, Composites Science and Technology, v. 66, pp. 1182-1189, 2006.

[21] RIEGEL, I.C., FREITAS, L.L., SAMIOS, D., "Envelhecimento físico de sistemas DGEBA/DDM investigado por análise térmica (DSC/DMA)”, Polímeros: Ciência e Tecnologia, v. 9, pp. 58-64, 1999.

[22] COSTA, M.L., ALMEIDA, S.F.M., REZENDE, M.C., "Resistência ao cisalhamento interlaminar de compósitos com resina epóxi com diferentes arranjos das fibras na presença de vazios", Polímeros: Ciência e Tecnologia, v. 11, pp. 182-189, 2001.

[23] COSTA, M.L., REZENDE, M.C, PARDINI, L.C., "Métodos de estudo da cinética de cura de resinas epóxi”, Polímeros: Ciência e Tecnologia, v. 2, pp. 37-44, 1999.

[24] ZAMAN, I., PHAN, T.T., KUAN, H-C., et al., "Epoxy/graphene platelets nanocomposites with two levels of interface strength", Polymer, v. 52, pp. 1603-1611, 2011.

[25] HU, H., WANG, X., WANGA, J., et al., "Preparation and properties of graphene nanosheetspolystyrene nanocomposites via in situ emulsion polymerization", Chemical Physics Letters, v. 484, pp. 247-253, 2010.

[26] SUAVE, J. Compósitos de epóxi com nanotubos de carbono de parede simples carboxilados: influência da adição de solventes e das condições de sonificação no processamento e nas propriedades, Dissertação de M.Sc., UDESC, Joinville, SC, Brasil, 2008.

[27] GRAYFER, E.D., NAZAROV, A.S., MAKOTCHENKO, V. G., KIM, S.-J., FEDOROV, V.E. Chemically modified graphene sheets by functionalization of highly exfoliated graphite, J. Mater. Chem., 2011,21, 3410-3414.

[28] KIM, C.-D., MIN, B.-K, JUNG, W.-S. Preparation of graphene sheets by the reduction of carbon monoxide, Carbon, Volume 47, Issue 6, May 2009, Pages 1610-1612. 